\title{
Design, Formulation and Evaluation of Transdermal Drug Delivery System of Budesonide
}

\author{
Updesh B. Lade*, Yogesh M. Amgaonkar, Rupesh V. Chikhale*, Dinesh M. Biyani, Milind J. Umekar
}

Department of Pharmaceutics, Smt. Kishoritai Bhoyar College of Pharmacy, Sadashivrao Patil Shikshan Sanstha, New Kamptee, Nagpur (M.S), India.

Email: *yamgaonkar123@gmail.com, rupeshchikhale7@gmail.com

Received January $24^{\text {th }}, 2011$; revised March $30^{\text {th }}, 2011$; accepted May $28^{\text {th }}, 2011$.

\begin{abstract}
Budesonide is a highly potent synthetic, nonhalogenated corticosteroid. The mechanism of action of corticosteroids in allergic rhinitis remains unknown, but may involve reductions in number of various mediator cells such as basophils, eosinophils, T-helper cells, mast cells, and neutrophils. In the nasal mucosa, nasal reactivity to allergens, and release of inflammatory mediators and proteolytic enzymes. Budesonide is very effective and quikly acting as it is rapidly and almost completely absorbed after oral administration, but has poor systemic availability (about 10\%) due to extensive first-pass metabolism in the liver, mainly by the cytochrome P450 isoenzyme CYP3A4. The major metabolites, 6- $\beta$ hydroxybudesonide and 16- $\alpha$-hydroxyprednisolone have less than $1 \%$ of the glucocorticoid activity of unchanged drug with a terminal half-life of about 2 - 4 hours. Polymeric films containing Eudragit RL 100: Eudragit RS: drug (7:3:1, 7: 2:1) and Ethyl cellulose: PVP: drug (7:3:1, 7:2:1) were selected for transdermal administration based on evaluation studies. These polymeric films were prepared by mercury substrate method employing PEG-400 as plasticizer. Two different penetration enhancers Urea and Dimethyl sulphoxide (DMSO) were employed in the study. The patches in each group were uniform in drug content, thickness. In Vitro drug permeation, moisture absorption and WVTR studies were carried out on these test patches. It was found that at all humidity condition the absorption increases which were linear to the moisture absorbed. In PVA and EUDRAGIT RL 100 patches the water vapor transmission rate was found to be higher at $75 \%$ RH, RT conditions. Therefore at both \% RH, RT condition the PVA and EUDRAGIT RL 100 patches provide the best resistance to water vapor. Therefore, when applied to animals (in further studies) these patches may provide more occlusion to water vapor loss from skin thus making atmosphere beneath the skin more humid that aid in drug permeation.
\end{abstract}

Keywords: Budesonide, Transdermal Drug Delivery

\section{Introduction}

Corticosteroids and their biologically active synthetic derivatives differ in their metabolic (glucocorticoid) and electrolyte-regulating (mineralocorticoid) activities. These agents are employed at physiological doses for replacement therapy when endogenous production is impaired. In addition, glucocorticoids potently suppress inflammation, and their use in a variety of inflammatory, asthmatic conditions, skin disorders, rhinitis, inflammatory bowel disease, collagenous colitis and autoimmune diseases makes them among the most frequently prescribed classes of drugs [1]. Budesonide is a highly potent synthetic, nonhalogenated corticosteroid. It has high glucocorticoid and weak mineralocorticoid activity [2]. Exact mechanism(s) of action of corticosteroids in allergic rhinitis remains unknown, but may involve reductions in the following: number of mediator cells (basophils, eosinophils, T-helper cells, mast cells, and neutrophils) in the nasal mucosa, nasal reactivity to allergens, and release of inflammatory mediators and proteolytic enzymes [3].

Budesonide is rapidly and almost completely absorbed after oral administration, but has poor systemic availability (about 10\%) due to extensive first-pass metabolism in the liver, mainly by the cytochrome P450 isoenzyme CYP3A4 [4]. The major metabolites, 6- $\beta$-hydroxybudesonide and $16-\alpha$-hydroxyprednisolone have less than $1 \%$ of the glucocorticoid activity of unchanged drug with a terminal half-life of about 2 - 4 hours [4-6].

The polymeric film containing Eudragit RL 100: Eudragit RS: drug (7:3:1, 7:2:1) and Ethyl cellulose: PVP: 
drug $(7: 3: 1,7: 2: 1)$ were selected for transdermal administration based on evaluation studies $[7,8]$. The polymeric films were prepared by mercury substrate method employing PEG-400 as plasticizer. Two different penetration enhancers Urea and Dimethyl sulphoxide (DMSO) were employed in the study. The dried polymeric film was evaluated using different parameters including thickness uniformity, drug content of the film, in vitro drug release from films and in vitro skin permeation of drug, prior to their in vivo evaluation $[8,9]$.

\section{Materials and Methods}

\subsection{Materials}

Budesonide (gift sample from M/s. Cipla Pharmaceuticals Ltd., Mumbai, India), Eudragit RL-100, Eudragit RS-100 (Röhm GmbH \& Co. KG, Pharma Polymers, Darmstadt, Germany), Polyethylene glycol (PEG-400), Cellophane Membrane, Polyvinyl alcohol (PVA), Ethyl cellulose (14 cps), PVP (Mol. Wt. 40,000), Potassium dihydrogenorthophosphate, Potassium carbonate, Potassium nitrate, Sodium chloride, Sodium hydroxide, Urea, Dimethyl sulphoxides (DMSO), Glyceryl triacetate, all other chemicals used were of analytical grade and obtained commercially.

\subsection{Animals}

The Swiss albino rats (170 - 190 gm) were obtained from National Chemical Labortary, Pune, India, and maintained at $25 \pm 1{ }^{\circ} \mathrm{C}$ for the study. The animals were housed in stainless metabolic cages and provided with standard diet and water ad libitum. Necessary approvals were obtained from CPCSEA India, for conducting the study.

\subsection{Preparation and Evaluation of Polymeric Films}

\subsubsection{Preparation of Transdermal Patch by Solvent Casting on Mercury Substrate [10,11]}

The transdermal patch was prepared by solvent evaporation technique on mercury substrate. Polymer solution was prepared in ethanol $(10 \mathrm{ml})$ and to it budesonide was added. The plasticizer or permeation enhancer or the pore forming agent were added during patch casting. The solution was poured on glass rings placed on mercury surface and allowed to dry in air for 24 hours. Circular patches of $2 \mathrm{~cm}$ diameter $\left(3.14 \mathrm{~cm}^{2}\right)$ were cut from semidried patches and placed in desiccator with $0 \%$ Relative Humidity (RH).

\subsubsection{Evaluation of patch}

1) Measurement of thickness [11]

Thickness was measured using micrometer screw gauge. Each patch was measured for thickness at five different points to ascertain thickness uniformity in patch.

2) Drug content [11]

Accurately weighed patches were individually dissolved in minimum quantity of ethanol and volume was made up to $20 \mathrm{ml}$ with $\mathrm{pH} 7.4$ phosphate buffer containing $2.5 \%$ ethanol. From this solution, $1 \mathrm{ml}$ was transferred to volumetric flask and volume was made up to 10 $\mathrm{ml}$. The absorbance was recorded at $247 \mathrm{~nm}$. The blank solution was prepared in the similar way except that the patches without drug were used.

3) Moisture absorption studies [12]

The moisture absorption study was carried out at 43 , $75,93 \% \mathrm{RH}, \mathrm{RT}$ at $25 \pm 1{ }^{\circ} \mathrm{C}$. The pre-weighed samples of patches were kept under the humidity conditions and weighed after 24 hours. The increase in weight indicates the moisture absorption by samples.

4) Dissolution studies $[13,14]$

The accurately weighed patches were fixed on glass discs of $2.5 \mathrm{~cm}$ diameter using standard glue. This assembly was kept in dissolution vessel such that the patch faced the dissolution media upwards. The dissolution media was $900 \mathrm{ml}$ of $\mathrm{pH} 7.4$ phosphate buffer containing $2.5 \%$ ethanol at $32 \pm 0.5^{\circ} \mathrm{C}$. The speed of paddle was kept at $50 \mathrm{rpm}$. Samples were withdrawn at 1 hour interval till 12 hours and replaced with the media. The absorbance was measured at $247 \mathrm{~nm}$ against the blank.

5) In vitro skin permeation studies [10]

Cellophane membrane was used. The membrane was mounted between the donor and receptor compartment of Franz diffusion cell. The patch was kept in contact with cellophane membrane. The receptor compartment contained $\mathrm{pH} 7.4$ phosphate buffer containing $2.5 \%$ ethanol. The assembly was kept on a magnetic stirrer and stirred at a speed of $200 \mathrm{rpm}$. The temperature of assembly was kept at $37 \pm 1{ }^{\circ} \mathrm{C}$. After each hour, $1 \mathrm{ml}$ of sample was withdrawn and replaced with same media up to 24 hours to study.

6) Effect of drugs loading and polymer concentration on film [15]

Films with different drug load and polymer concentration were prepared and studied for their thickness, moisture absorption, and In vitro drug permeation.

7) Effect of penetration enhancers on film

Two penetration enhancers namely urea, dimethylsulphoxide (DMSO) were incorporated in different proportion 5 and $10 \%$. The films were then evaluated for the properties as mentioned earlier.

8) Effect of plasticizers on film

Two plasticizers namely polyethylene glycol-400, glyceryl triacetate were added in different concentration 5 and $10 \%$ of polymer concentration. These films were then evaluated for the properties as mentioned earlier. 


\begin{tabular}{cc}
\hline Model & Equation \\
\hline Zero order Kinetics & $\mathrm{Q}=\mathrm{Q}_{0}-\mathrm{K}_{0} \mathrm{t}$ \\
First order kinetics & $\mathrm{Q}=\mathrm{Q}_{0}\left(1-\mathrm{e}^{-\mathrm{Klt}}\right)$ \\
Higuchi square root model & $\mathrm{Q}_{\mathrm{t}}=\mathrm{K}_{\mathrm{H}} \cdot \mathrm{t}^{1 / 2}$ \\
Hixson-Crowell cube root model & $\sqrt[3]{\mathrm{Q}_{0}-\sqrt[3]{\mathrm{Q}_{\mathrm{t}}}=\mathrm{K}_{\mathrm{HC}}}{ }^{\mathrm{t}}$ \\
Korsmeyer-peppas model & $\mathrm{Q}_{\mathrm{t}} / \mathrm{Q}_{\infty}=\mathrm{K}_{\mathrm{k}} \cdot \mathrm{t}^{\mathrm{n}}$ \\
\hline
\end{tabular}

where, Qt-amount of drug released at time $t$; Qo-initial amount of drug. and $\mathrm{Ko}, \mathrm{K} 1, \mathrm{KH}, \mathrm{KHC}$ and KK are the coefficients of equations.

9) Water vapor transmission rate (WVTR)

The WVTR study was carried out in desiccators main- tained at 43 and $75 \% \mathrm{RH}$ at $25 \pm 1{ }^{\circ} \mathrm{C}$ using saturated solution of potassium carbonate and sodium chloride respectively. Patches were placed on the mouth of glass vials containing fused calcium chloride and sealed using silicon wax. These vials were accurately weighed and placed in desiccators at $0 \% \mathrm{RH}$. The weight of these vi- als was recorded after 24 hours. The increase in weight was indicative of water transmission across the patch.

10) Study of drug release kinetics [16]

In order to investigate the drug release mechanism from patches, the \% cumulative drug release data was analyzed with following mathematical model.

The most appropriate model was selected on the basis of goodness of fit test. The zero order kinetic describes the systems in which the drug release rate is independent of its concentration. The drug releases slowly (assuming that the area does not change and no equilibrium conditions are obtained). The first order kinetics describes the systems in which drug release rate is concentration dependent. Higuchi model describes the release of watersoluble drug from an insoluble matrix as a diffusion process based on the Fick's law and is square root time dependent.

The Hixson-Crowell cube root law describes the drug release from a system depends upon the change in surface area or diameter of particle or system and involves no diffusion mechanism. Korsmeyer-Peppas model describes the fraction of drug release relates exponentially with respect to time. This model is generally used to analyze the release of pharmaceutical polymeric dosage forms, when the release mechanism is not well known or when more than one type of release phenomena could be involved.

11) Skin irritancy study $[17,18]$

Area on the back of rats was clean shaved 24 hours prior to testing. Optimize patch was applied to the clean area and kept in its place by adhesive tape. After every hour, the patch was removed and observed visually for signs of edema or erythema and scored according to Draize's scoring index. Patch without drug was used as control patch.

\section{Results and Discussion}

\subsection{Table 1. Effect of Drug Loading and Polymer Concentration on Film}

The patches in each group are uniform in drug distribution. The thickness and weight increases with the increase in polymer concentration. The films formed are transparent in appearance. (Tables 1 and 2)

\subsection{Table 2. Characterization of Eudragit RL 100 Patches}

\subsection{Moisture absorption study}

The patches were subjected to different \% RH, RT conditions and the absorption of moisture noted the results are shown as under;

\subsubsection{Table 3. Moisture Absorption of PVA Patches} Visual examination indicates that, at $43 \% \mathrm{RH}$, RT no change was observed in PVA patches on 7th day, while at $75 \% \mathrm{RH}$, RT the PVA Patches lost there shape on 7 th

Table 1. Effect of drug loading and polymer concentration on film.

\begin{tabular}{|c|c|c|c|c|c|c|c|}
\hline PVA & $\begin{array}{l}\text { Eudragit } \\
\text { RL } 100\end{array}$ & $\begin{array}{c}\text { \% Polymer } \\
\text { Concentra- } \\
\text { tion }\end{array}$ & Drug & $\begin{array}{l}\text { Appear- } \\
\text { ance }\end{array}$ & $\begin{array}{c}\text { Thick- } \\
\text { ness } \\
(\mathrm{mm})\end{array}$ & $\begin{array}{c}\text { Weight } \\
\text { (mg) }\end{array}$ & $\begin{array}{l}\text { Con- } \\
\text { tent } \\
\text { (mg) }\end{array}$ \\
\hline P1 & U1 & 5 & - & Transparent & 0.019 & 22.1 & 1.910 \\
\hline $\mathbf{P} 2$ & $\mathrm{U} 2$ & 6 & - & ---Do---- & 0.020 & 24.4 & 1.877 \\
\hline P3 & U3 & 7 & - & ---Do---- & 0.021 & 29.1 & 1.891 \\
\hline P4 & U4 & 8 & - & ---Do---- & 0.023 & 29.5 & 1.848 \\
\hline V1 & Eu1 & 5 & 15 & ---Do---- & 0.015 & 18.6 & 1.040 \\
\hline V2 & Eu2 & 6 & 15 & ---Do---- & 0.017 & 20.2 & 1.002 \\
\hline V3 & Eu3 & 7 & 15 & ---Do---- & 0.019 & 23.9 & 1.035 \\
\hline V4 & Eu4 & 8 & 15 & ---Do---- & 0.021 & 26.4 & 0.988 \\
\hline F1 & El1 & 5 & 10 & ---Do---- & 0.018 & 16.2 & 0.137 \\
\hline F2 & E12 & 6 & 10 & ---Do---- & 0.019 & 22.1 & 0.146 \\
\hline F3 & E13 & 7 & 10 & ---Do---- & 0.021 & 25.4 & 0.170 \\
\hline F4 & E14 & 8 & 10 & ---Do---- & 0.023 & 29.2 & 0.212 \\
\hline J1 & Eb1 & 5 & 5 & Transparent & 0.019 & 22.1 & 1.910 \\
\hline $\mathbf{J} 2$ & $\mathrm{~Eb} 2$ & 6 & 5 & ---Do---- & 0.020 & 24.4 & 1.877 \\
\hline J3 & Eb3 & 7 & 5 & ---Do---- & 0.021 & 29.1 & 1.891 \\
\hline $\mathrm{J} 4$ & $\mathrm{~Eb} 4$ & 8 & 5 & ---Do---- & 0.023 & 29.5 & 1.848 \\
\hline
\end{tabular}

Table 2. Characterization of Eudragit RL 100 patches.

\begin{tabular}{|c|c|c|c|c|}
\hline Code & Appearance & Thickness & $\begin{array}{c}\text { Weight } \\
\text { (Mg) }\end{array}$ & $\begin{array}{c}\text { Content } \\
\text { (Mg) }\end{array}$ \\
\hline Eu1 & Transparent & 0.019 & 21.8 & 2.671 \\
\hline Eu2 & ---Do---- & 0.021 & 23.8 & 2.713 \\
\hline Eu3 & ---Do---- & 0.023 & 26.4 & 2.652 \\
\hline Eu4 & ---Do---- & 0.025 & 29.7 & 2.661 \\
\hline El1 & ---Do---- & 0.017 & 24.8 & 1.271 \\
\hline El2 & ---Do---- & 0.018 & 25.6 & 1.290 \\
\hline El3 & ---Do---- & 0.019 & 29.8 & 1.281 \\
\hline El4 & ---Do---- & 0.022 & 32.1 & 1.267 \\
\hline EB1 & ---Do---- & 0.015 & 19.8 & 0.165 \\
\hline EB2 & ---Do---- & 0.017 & 22.4 & 0.174 \\
\hline EB3 & ---Do---- & 0.020 & 27.3 & 0.151 \\
\hline EB4 & ---Do---- & 0.023 & 28.6 & 0.156 \\
\hline
\end{tabular}


Table 3. Moisture absorption of PVA patches.

\begin{tabular}{|c|c|c|c|c|c|c|c|c|c|}
\hline \multirow{2}{*}{ CODE } & \multicolumn{3}{|c|}{$\begin{array}{c}43 \% \text { RH, RT } \\
\text { Pot. Carbonate } \\
\end{array}$} & \multicolumn{3}{|c|}{$\begin{array}{c}\text { 75\% RH, RT } \\
\text { Sod. Chloride } \\
\end{array}$} & \multicolumn{3}{|c|}{$\begin{array}{c}\text { 93\% RH, RT } \\
\text { Pot. nitrate }\end{array}$} \\
\hline & $\begin{array}{l}\text { Wt. of patch } \\
\text { (mg) }\end{array}$ & $\begin{array}{l}\text { Moist. Absorp. } \\
\text { (mg) }\end{array}$ & $\%$ ABS. & $\begin{array}{l}\text { Wt. of patch } \\
\text { (mg) }\end{array}$ & $\begin{array}{l}\text { Moist. Absorp. } \\
\text { (mg) }\end{array}$ & $\%$ ABS & $\begin{array}{l}\text { Wt. of patch } \\
\text { (mg) }\end{array}$ & $\begin{array}{l}\text { Moist. Absorp. } \\
\text { (mg) }\end{array}$ & $\%$ ABS \\
\hline $\mathrm{P} 2$ & 18.5 & 1.5 & 8.10 & 17.9 & 2.9 & 16.20 & 17.0 & 6.1 & 35.88 \\
\hline P3 & 17.9 & 0.4 & 2.23 & 18.0 & 3.6 & 20.00 & 16.0 & 8.4 & 52.50 \\
\hline $\mathrm{P} 4$ & 24.9 & 1.4 & 5.62 & 24.7 & 3.9 & 15.78 & 19.8 & 9.2 & 46.46 \\
\hline V1 & 22.1 & 10.5 & 47.51 & 21.9 & 13.8 & 63.01 & 22.4 & 17.0 & 75.89 \\
\hline $\mathrm{V} 2$ & 24.4 & 7.14 & 29.26 & 24.6 & 15.8 & 64.22 & 25.2 & 19.0 & 75.39 \\
\hline V3 & 29.1 & 5.79 & 19.89 & 28.2 & 8.2 & 29.07 & 27.4 & 13.7 & 50.00 \\
\hline V4 & 29.5 & 7.15 & 24.23 & 30.4 & 11.1 & 36.51 & 29.6 & 15.6 & 52.70 \\
\hline F1 & 18.6 & 11.17 & 60.05 & 18.8 & 6.7 & 35.63 & 19.4 & 7.6 & 39.17 \\
\hline $\mathrm{F} 2$ & 20.2 & 6.29 & 31.13 & 19.9 & 8.75 & 43.96 & 20.9 & 10.6 & 51.45 \\
\hline F3 & 23.9 & 7.43 & 31.08 & 24.2 & 8.55 & 35.33 & 23.6 & 10.8 & 45.76 \\
\hline F4 & 26.4 & 8.03 & 30.41 & 27.0 & 10.10 & 37.40 & 27.2 & 14.3 & 52.57 \\
\hline $\mathrm{J} 1$ & 16.2 & 4.1 & 25.3 & 16.5 & 4.9 & 29.69 & 16.9 & 5.6 & 33.13 \\
\hline $\mathrm{J} 3$ & 25.4 & 2.4 & 9.44 & 24.3 & 8.6 & 35.39 & 25.9 & 9.9 & 38.22 \\
\hline $\mathrm{J} 4$ & 29.2 & 2.8 & 9.65 & 28.2 & 7.0 & 24.82 & 29.6 & 9.0 & 30.40 \\
\hline
\end{tabular}

Table 4. Moisture absorption of Eudragit RL 100 patches.

\begin{tabular}{|c|c|c|c|c|c|c|c|c|c|}
\hline \multirow{2}{*}{ CODE } & \multicolumn{3}{|c|}{$\begin{array}{c}43 \% \text { RH, RT } \\
\text { Pot. Carbonate }\end{array}$} & \multicolumn{3}{|c|}{$\begin{array}{c}\text { 75\% RH, RT } \\
\text { Sod. Chloride } \\
\end{array}$} & \multicolumn{3}{|c|}{$\begin{array}{c}\text { 93\% RH, RT } \\
\text { Pot. nitrate }\end{array}$} \\
\hline & $\begin{array}{c}\text { Wt. of } \\
\text { patch (mg) }\end{array}$ & $\begin{array}{l}\text { Moist. Absorp. } \\
\text { (mg) }\end{array}$ & $\%$ ABS. & $\begin{array}{c}\text { Wt. of } \\
\text { patch (mg) }\end{array}$ & $\begin{array}{l}\text { Moist. Absorp. } \\
\text { (mg) }\end{array}$ & $\%$ ABS & $\begin{array}{c}\text { Wt. of } \\
\text { patch (mg) }\end{array}$ & $\begin{array}{l}\text { Moist. Absorp. } \\
\text { (mg) }\end{array}$ & $\% \mathrm{ABS}$ \\
\hline U1 & 15.9 & 0.3 & 1.88 & 15.3 & 0.3 & 1.96 & 15.6 & 0.6 & 3.84 \\
\hline U2 & 17.7 & 2.0 & 11.29 & 17.9 & 1.4 & 7.82 & 17.4 & 2.0 & 11.49 \\
\hline U3 & 18.8 & 1.5 & 7.97 & 18.1 & 1.3 & 7.18 & 18.9 & 1.2 & 6.34 \\
\hline U4 & 20.1 & 0.8 & 3.98 & 20.7 & 0.5 & 2.41 & 20.4 & 1.6 & 7.84 \\
\hline EU1 & 21.8 & 0.4 & 1.83 & 22.2 & 1.3 & 5.85 & 21.3 & 1.4 & 6.57 \\
\hline EU2 & 23.8 & 2.9 & 12.18 & 23.6 & 0.5 & 2.11 & 23.2 & 3.3 & 14.22 \\
\hline EU3 & 26.4 & 0.7 & 2.65 & 27.1 & 0.8 & 2.95 & 26.8 & 12.5 & 9.32 \\
\hline EU4 & 29.7 & 1.8 & 6.06 & 29.9 & 2.8 & 9.36 & 30.1 & 0.6 & 1,99 \\
\hline EL1 & 24.8 & 1.2 & 4.83 & 24.3 & 0.8 & 3.29 & 21.7 & 2.5 & 11.52 \\
\hline EL2 & 25.6 & 1.4 & 5.46 & 25.1 & 0.6 & 2.39 & 26.1 & 3.5 & 13.40 \\
\hline EL3 & 29.8 & 0.6 & 2.01 & 29.3 & 1.9 & 6.48 & 28.6 & 2.6 & 9.09 \\
\hline EL4 & 32.1 & 1.6 & 4.98 & 31.8 & 1.3 & 4.08 & 32.7 & 2.8 & 7.95 \\
\hline EB1 & 19.8 & 0.9 & 4.54 & 19.6 & 0.4 & 2.04 & 20.1 & 3.0 & 14.92 \\
\hline EB2 & 22.4 & 1.0 & 4.46 & 21.9 & 0.6 & 2.73 & 22.9 & 1.4 & 6.11 \\
\hline EB3 & 27.3 & 1.4 & 5.12 & 27.8 & 1.1 & 3.95 & 27.0 & 1.5 & 5.55 \\
\hline EB4 & 28.6 & 0.8 & 2.79 & 28.3 & 0.7 & 2.47 & 28.9 & 1.8 & 6.22 \\
\hline
\end{tabular}

day, however the patches were found to be less stable at 93\% RH, RT on 5 th day.

\subsubsection{Table 4. Moisture Absorption of Eudragit RL 100 Patches}

For Eudragit RL100 patches at 43\% RH, RT the Patches lost their shape on 7 th day, while at $75 \%$ RH, RT the Patches lost their shape on 5th day and at 93\% RH, RT patch were still more unstable and lost their shape at 4 th day.

Since the absorption pattern is not uniform in both the cases therefore no conclusion can be drawn regarding the stability of patch from the above data. At every \% RH, RT condition the Eudragit RL 100 patches absorb less moisture than PVA patches, but PVA patches were found to be more stable than the Eudragit RL 100 patches.

\subsection{In Vitro Drug Permeation through Cellophane Membrane}

The in-vitro permeation of PVA patches was studied by using cellophane membrane, results obtained are as shown under;

3.4.1. Table 5. Drug Permeation from PVA Patches (15 $\mathrm{mg}$ )

3.4.2. Table 6. Drug Permeation from PVA Patches (10 $\mathrm{mg}$ )

3.4.3. Table 7. Drug Permeation from PVA Patches (5 $\mathrm{mg})$

3.4.4. Figure 1. Effect of PVA Concentration with Constant Drug Concentration (15 mg) on Drug Permeation 
Table 5. Drug permeation from PVA patches (15 mg).

\begin{tabular}{ccccccccc}
\hline & \multicolumn{7}{c}{ (\%) Percent permeated } \\
\cline { 2 - 9 } CODE & \multicolumn{7}{c}{ Time in (hrs.) } \\
\cline { 2 - 9 } & 1 & 2 & 3 & 4 & 5 & 6 & 12 & 24 \\
\hline V1 & 4.11 & 4.98 & 6.21 & 7.53 & 8.85 & 10.33 & 11.39 & 12.98 \\
V2 & 4.39 & 5.30 & 6.54 & 7.79 & 9.06 & 10.72 & 11.94 & 13.48 \\
V3 & 4.99 & 6.16 & 7.48 & 8.73 & 10.15 & 11.58 & 12.97 & 15.05 \\
V4 & 4.19 & 5.14 & 6.36 & 7.64 & 8.98 & 10.26 & 11.37 & 12.89 \\
\hline
\end{tabular}

Table 6. Drug permeation from PVA patches $(10 \mathrm{mg})$.

\begin{tabular}{ccccccccc}
\hline & \multicolumn{7}{c}{ (\%) Percent permeated } \\
\cline { 2 - 9 } CODE & \multicolumn{7}{c}{ Time in (hrs.) } \\
\cline { 2 - 9 } & 1 & 2 & 3 & 4 & 5 & 6 & 12 & 24 \\
\hline F1 & 3.82 & 5.22 & 6.27 & 7.31 & 8.29 & 9.26 & 10.72 & 12.06 \\
F2 & 4.19 & 5.61 & 7.19 & 8.32 & 9.54 & 10.70 & 12.14 & 13.43 \\
F3 & 4.79 & 5.88 & 7.56 & 8.94 & 10.18 & 11.71 & 13.14 & $\mathbf{1 4 . 8 6}$ \\
F4 & 3.63 & 5.11 & 5.93 & 7.14 & 8.16 & 9.36 & 11.06 & 12.41 \\
\hline
\end{tabular}

Table 7. Drug permeation from PVA patches $(5 \mathrm{mg})$.

\begin{tabular}{ccccccccc}
\hline & \multicolumn{8}{c}{ (\%) Percent permeated } \\
\cline { 2 - 9 } CODE & \multicolumn{7}{c}{ Time in (hrs.) } \\
\cline { 2 - 9 } & 1 & 2 & 3 & 4 & 5 & 6 & 12 & 24 \\
\hline $\mathrm{J} 1$ & 3.42 & 4.81 & 5.83 & 6.85 & 7.82 & 8.77 & 10.21 & 11.53 \\
$\mathrm{~J} 2$ & 3.79 & 5.20 & 6.76 & 7.87 & 9.07 & 10.21 & 11.63 & 12.91 \\
$\mathrm{~J} 3$ & 4.40 & 5.46 & 7.13 & 8.49 & 9.71 & 11.21 & 12.63 & $\mathbf{1 4 . 3 2}$ \\
$\mathrm{J} 4$ & 3.23 & 4.71 & 5.48 & 6.68 & 8.48 & 9.84 & 11.34 & 12.57 \\
\hline
\end{tabular}

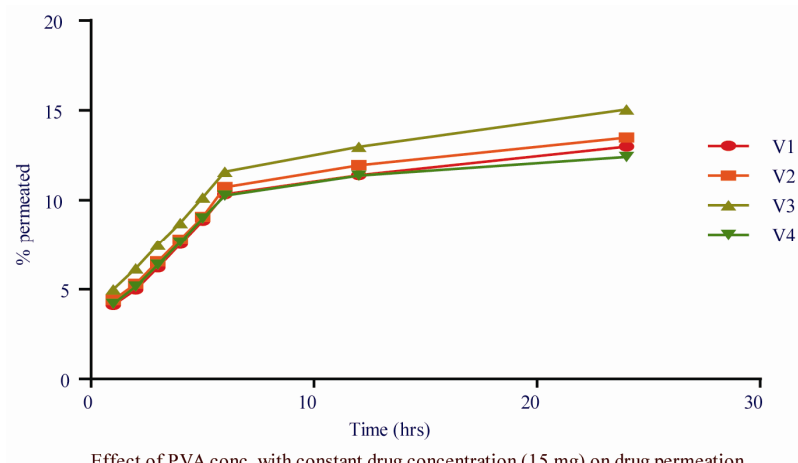

Figure 1. Effect of PVA concentration with constant drug concentration (15 $\mathrm{mg}$ ) on drug permeation.

3.4.5. Figure 2. Effect of PVA Concentration with Constant Drug Concentration (10 mg) on Drug Permeation

\subsubsection{Figure 3. Effect of PVA Concentration with Constant Drug Concentration (5 mg) on Drug Permeation}

The in vitro drug permeation in all the cases increase up to $7 \% \mathrm{w} / \mathrm{w}$ polymer concentration while at $8 \%$ it decreases with constant drug load, the permeation of drug increases with increase in polymer content up to $7 \% \mathrm{w} / \mathrm{w}$ and thereafter it decrease. With constant polymer concentration, higher drug load gives higher permeation of drug. Thus the maximum percent permeation in $\mathrm{V}, \mathrm{F}$,

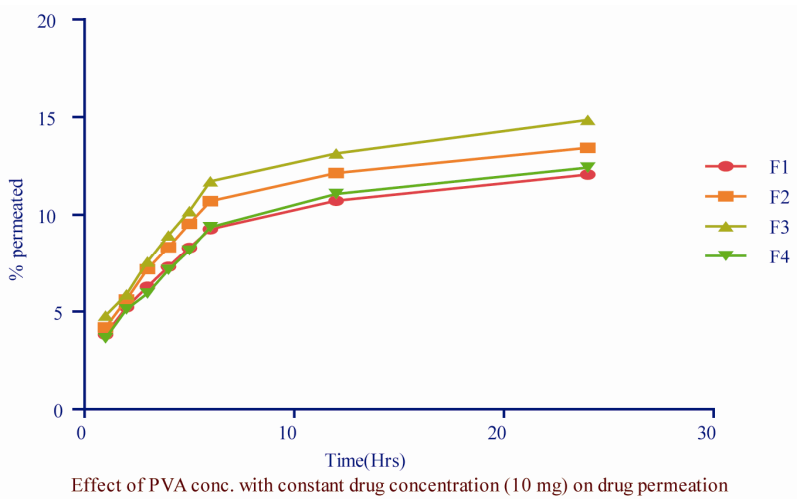

Figure 2. Effect of PVA concentration with constant drug concentration $(10 \mathrm{mg})$ on drug permeation.

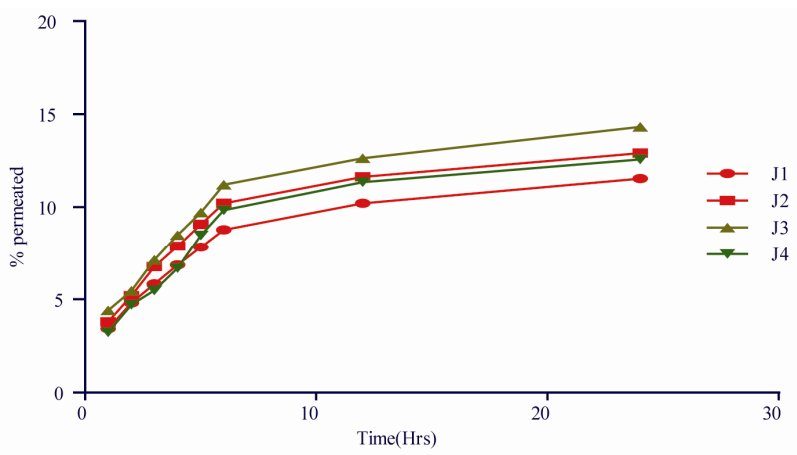

Effect of PVA conc. with constant drug concentration $(5 \mathrm{mg})$ on drug permeation

Figure 3. Effect of PVA concentration with constant drug concentration ( $5 \mathrm{mg}$ ) on drug permeation.

and $\mathrm{J}$ series was observed with $7 \% \mathrm{w} / \mathrm{w}$ polymer concentration and it was $15.05 \%, 14.86 \%, 14.32 \%$ respectively as represented by Figure 1, 2 and 3 for PVA concentration.

\subsection{In Vitro Drug Permeation through Cellophane Membrane}

The drug permeation through cellophane membrane was studied, results obtained are as shown under;

3.5.1. Table 8. Drug Permeation from EUDRAGIT RL 100 Patches $(15 \mathrm{mg})$

3.5.2. Figure 4. Effect of EUDRAGIT RL 100 conc. with Constant Drug Concentration (15 mg) on Drug Permeation

3.5.3. Table 9. Drug Permeation from EUDRAGIT RL 100 Patches $(10 \mathrm{mg})$

3.5.4. Figure 5. Effect of EUDRAGIT RL 100 conc. with Constant Drug Concentration $(10 \mathrm{mg}$ ) on Drug Permeation 
Table 8. Drug permeation from EUDRAGIT RL 100 patches (15 mg).

\begin{tabular}{ccccccccc}
\hline & \multicolumn{7}{c}{ (\%) Percent permeated } \\
\cline { 2 - 9 } CODE & $\mathbf{1}$ & $\mathbf{2}$ & $\mathbf{3}$ & $\mathbf{4}$ & $\mathbf{5}$ & $\mathbf{6}$ & $\mathbf{1 2}$ & $\mathbf{2 4}$ \\
\cline { 2 - 8 } & $\mathbf{1}$ & $\mathbf{8}$ & $\mathbf{7}$ \\
\hline EU1 & 5.76 & 6.61 & 7.83 & 8.74 & 10.25 & 11.49 & 13.06 & 14.60 \\
EU2 & 6.23 & 7.46 & 8.55 & 9.85 & 10.85 & 12.13 & 13.46 & $\mathbf{1 5 . 4 4}$ \\
EU3 & 5.28 & 6.54 & 7.92 & 8.89 & 10.48 & 11.48 & 13.15 & 14.72 \\
EU4 & 5.45 & 6.41 & 7.54 & 8.56 & 9.87 & 11.11 & 12.37 & 13.92 \\
\hline
\end{tabular}

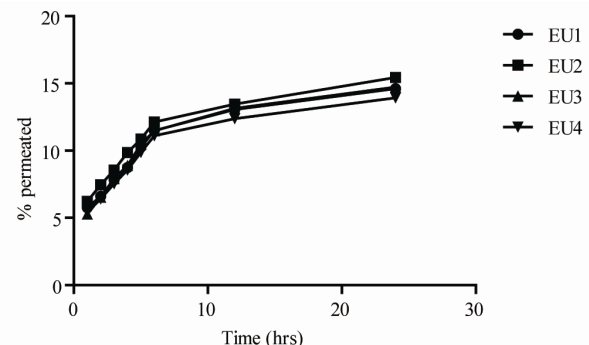

Effect of EUDRAGIT RL 100 conc. with constant drug concentration (15 mg) on drug permeation.

Figure 4. Effect of EUDRAGIT RL 100 conc. with constant drug concentration (15 mg) on drug permeation.

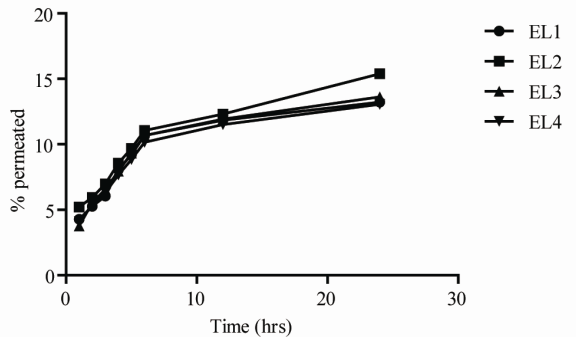

Effect of EUDRAGIT RL 100 conc. with constant drug concentration $(10 \mathrm{mg}$ ) on drug permeation.

Figure 5. Effect of EUDRAGIT RL 100 conc. with constant drug concentration $(10 \mathrm{mg})$ on drug permeation.

Table 9. Drug permeation from EUDRAGIT RL 100 patches $(10 \mathrm{mg})$.

\begin{tabular}{ccccccccc}
\hline & \multicolumn{7}{c}{ Time in (hrs) } \\
\cline { 2 - 9 } CODE & \multicolumn{7}{c}{ Percent permeated } \\
\cline { 2 - 8 } & $\mathbf{1}$ & $\mathbf{2}$ & $\mathbf{3}$ & $\mathbf{4}$ & $\mathbf{5}$ & $\mathbf{6}$ & $\mathbf{1 2}$ & $\mathbf{2 4}$ \\
\hline EL1 & 4.28 & 5.25 & 6.44 & 8.05 & 9.21 & 10.68 & 11.84 & 13.22 \\
EL2 & 5.21 & 5.94 & 6.97 & 8.56 & 9.69 & 11.05 & 12.3 & $\mathbf{1 5 . 3 9}$ \\
EL3 & 3.76 & 5.54 & 6.56 & 7.94 & 9.32 & 10.67 & 11.92 & 13.63 \\
EL4 & 4.09 & 5.32 & 6.33 & 7.65 & 8.84 & 10.16 & 11.50 & 13.06 \\
\hline
\end{tabular}

3.5.5. Table 10. Drug Permeation from EUDRAGIT RL $100(5 \mathrm{mg})$

\subsubsection{Figure 6. Effect of EUDRAGIT RL 100 conc. with Constant Drug Concentration (5 mg) on Drug Permeation}

The in vitro drug permeation in all the cases increase upto $6 \% \mathrm{w} / \mathrm{w}$ polymer concentration \& there after it decreases. Higher drug load gives higher permeation of drug. thus the maximum percent permeation in EU2, EL2, EB2 series was observed with $6 \% \mathrm{w} / \mathrm{w}$ polymer
Table 10. Drug Permeation from EUDRAGIT RL 100 (5 mg).

\begin{tabular}{ccccccccc}
\hline & \multicolumn{7}{c}{ (\%) Percent permeated } \\
\cline { 2 - 9 } CODE & \multicolumn{7}{c}{ Time in (hrs) } \\
\cline { 2 - 9 } & $\mathbf{1}$ & $\mathbf{2}$ & $\mathbf{3}$ & $\mathbf{4}$ & $\mathbf{5}$ & $\mathbf{6}$ & $\mathbf{1 2}$ & $\mathbf{2 4}$ \\
\hline EB1 & 3.89 & 5.11 & 6.01 & 7.60 & 8.74 & 10.66 & 11.82 & 13.21 \\
EB2 & 4.82 & 5.53 & 6.54 & 8.11 & 9.22 & 11.26 & 13.15 & $\mathbf{1 5 . 2 0}$ \\
EB3 & 3.37 & 5.13 & 6.13 & 7.49 & 8.86 & 10.42 & 11.66 & 13.60 \\
EB4 & 3.70 & 4.91 & 5.90 & 7.20 & 8.37 & 9.76 & 11.42 & 12.67 \\
\hline
\end{tabular}

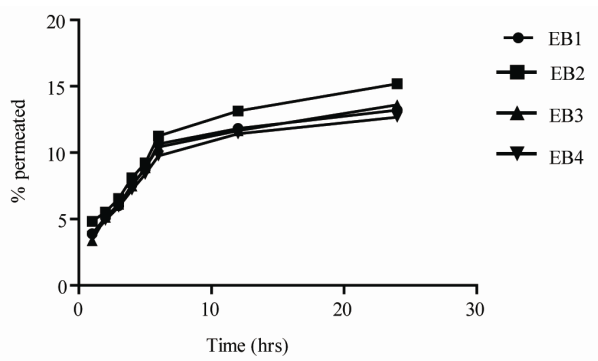

Effect of EUDRAGIT RL 100 conc. with constant drug concentration $(5 \mathrm{mg})$ on drug permeation

Figure 6. Effect of EUDRAGIT RL 100 conc. with constant drug concentration (5 mg) on drug permeation.

concentration and it was $15.05 \%, 14.86 \%, 14.32 \%$ respectively after $24 \mathrm{hrs}$. PVA $7 \% \mathrm{w} / \mathrm{w}$ gives maximum drug permeation while EUDRAGIT RL 100 at $6 \% \mathrm{w} / \mathrm{w}$ gives maximum permeation of drug. The maximum \% permeation with PVA was 15.05 while with EUDRAGIT RL 100 it was $15.44 \%$. For same drug content, the permeation through EUDRAGIT RL100 was better and higher than with PVA. Also with constant polymer concentration the drug permeation by EUDRAGIT RL100 was higher than PVA.

Therefore, with EUDRAGIT RL 100 better permeation may be obtained with less polymer requirement in in-vitro studies, therefore, EUDRAGIT RL 100 was better polymer than PVA.

\subsection{Effect of Penetration Enhancer on Film}

The compositions of various films and patches obtained were examined and characterized for the parameter are shown as below;

3.6.1. Table 11. Compositions of Patches with Different Penetration Enhancers.

\subsubsection{Table 12. Characterization of PVA \& EUDRAGIT RL 100 with Different Penetration Enhancers}

In both the cases the patches formed are uniform with respect to drug content also with increase in the amount of penetration enhancer the weight of the patch also increase linearly. The thickness was also to be uniform throughout the patch. 
Table 11. Compositions of patches with different penetration enhancers.

\begin{tabular}{|c|c|c|c|c|}
\hline CODE & Polymer (mg) & Drug (mg) & Urea (mg) & DMSO (mg) \\
\hline S1/M1 & $\begin{array}{l}\text { PVA/Eudragit } \\
\text { RL100 - } 700\end{array}$ & 5 & 35 & -- \\
\hline $\mathrm{S} 2 / \mathrm{M} 2$ & $\begin{array}{l}\text { PVA/Eudragit } \\
\text { RL100 - } 700\end{array}$ & 5 & 70 & -- \\
\hline $\mathrm{T} 1 / \mathrm{R} 1$ & $\begin{array}{l}\text { PVA/Eudragit } \\
\text { RL100 - } 700\end{array}$ & 5 & -- & 35 \\
\hline $\mathrm{T} 2 / \mathrm{R} 2$ & $\begin{array}{l}\text { PVA/Eudragit } \\
\text { RL100 - } 700\end{array}$ & 5 & -- & 70 \\
\hline
\end{tabular}

Table 12. Characterization of PVA \& EUDRAGIT RL 100 with different penetration enhancers.

\begin{tabular}{ccccc}
\hline CODE & Appearance & $\begin{array}{c}\text { Thickness } \\
\text { (mm) }\end{array}$ & Weight (mg) $\begin{array}{c}\text { Drug con- } \\
\text { tent (mg) }\end{array}$ \\
\hline S1 & Transparent & 0.018 & 26.3 & 0.302 \\
M1 & Transparent & 0.017 & 24.3 & 0.472 \\
S2 & Transparent & 0.020 & 29.1 & 0.453 \\
M2 & Transparent & 0.018 & 27.9 & 0.879 \\
T1 & Transparent & 0.017 & 28.2 & 0.226 \\
R1 & Transparent & 0.019 & 26.2 & 0.335 \\
T2 & Transparent & 0.019 & 32.1 & 0.430 \\
R2 & Transparent & 0.021 & 29.4 & 0.789 \\
\hline
\end{tabular}

Table 13. Moisture Absorption by PVA and EUDRAGIT RL 100 Patches with Different Penetration Enhancers.

\begin{tabular}{|c|c|c|c|c|c|c|c|c|c|}
\hline \multirow[b]{2}{*}{ CODE } & \multicolumn{3}{|c|}{ 43\% RH, RT } & \multicolumn{3}{|c|}{ 75\% RH, RT } & \multicolumn{3}{|c|}{ 93\% RH, RT } \\
\hline & $\begin{array}{l}\text { Wt. of Patch } \\
\text { (Mg) }\end{array}$ & $\begin{array}{l}\text { Moist. Ab- } \\
\text { sor. (Mg) }\end{array}$ & \% Absorption & $\begin{array}{l}\text { Wt. of Patch } \\
\text { (Mg) }\end{array}$ & $\begin{array}{c}\text { Moist. Ab- } \\
\text { sor. (Mg) }\end{array}$ & \%Absorption & $\begin{array}{l}\text { Wt. of Patch } \\
\text { (Mg) }\end{array}$ & $\begin{array}{c}\text { Moist. Ab- } \\
\text { sor. (Mg) }\end{array}$ & \% Absorption \\
\hline S1 & 26.1 & 4.5 & 17.24 & 27.2 & 11.9 & 43.75 & 28.0 & 15.0 & 53.57 \\
\hline $\mathrm{S} 2$ & 28.7 & 6.2 & 21.60 & 28.5 & 14.1 & 49.47 & 28.3 & 16.8 & 59.36 \\
\hline $\mathrm{T} 1$ & 28.0 & 3.1 & 11.07 & 28.3 & 7.4 & 26.14 & 27.9 & 11.2 & 40.14 \\
\hline $\mathrm{T} 2$ & 31.7 & 7.1 & 22.39 & 31.6 & 11.8 & 37.34 & 32.0 & 14.6 & 45.62 \\
\hline M1 & 24.6 & 2.1 & 8.53 & 24.8 & 3.9 & 15.72 & 24.2 & 4.8 & 19.83 \\
\hline M2 & 27.4 & 3.6 & 13.13 & 27.8 & 5.4 & 19.42 & 27.9 & 6.7 & 24.01 \\
\hline $\mathrm{R} 1$ & 26.4 & 1.8 & 6.8 & 26.6 & 2.6 & 9.77 & 26.8 & 3.5 & 13.05 \\
\hline $\mathrm{R} 2$ & 29.2 & 2.9 & 9.93 & 29.4 & 3.7 & 12.58 & 29.5 & 5.1 & 17.22 \\
\hline
\end{tabular}

\subsection{Moisture Absorption}

\subsubsection{Table 13. Moisture Absorption by PVA and EUDRAGIT RL 100 Patches with Different Penetration Enhancers}

At $43 \%$ RH, RT, the moisture absorption by PVA patches is comparable with patch without the enhancers i.e. J3. The moisture absorption increases with increase in enhancer content. DMSO $10 \% \mathrm{w} / \mathrm{w}$ gives greater $\mathrm{ab}-$ sorption than other i.e. $22.39 \%$. It is found that $75 \% \mathrm{RH}$, RT the absorption pattern is higher than at $43 \% \mathrm{RH}, \mathrm{RT}$. At $75 \% \mathrm{RH}$, RT maximum absorption is shown by urea $10 \%$ w/w with $49.47 \%$ absorption \& at $95 \%$ RH, RT with $59.36 \%$. All humidity condition the absorption increases with increase in enhancer content. As the humidity increases, there increase in moisture absorption and this increase linear. However, at 43\% RH, RT all the patches retain their shape at the end of 7 th day, At $75 \%$ RH, RT the urea patches lose their shape on 7 th day, while DMSO patches a little sticky to touch. At 95\% RH, RT the urea patches lose their shape on 4th day while DMSO patches still sticky \& lose their shape on 6th day.

It is found that around $43 \% \mathrm{RH}, \mathrm{RT}$, the moisture absorption by EUDRAGIT RL 100 patches is comparable with patch without the enhancers i.e. EB3 urea 10\% w/w absorb moisture more than any other. When the conditions were of $75 \% \mathrm{RH}, \mathrm{RT}$ and at $95 \% \mathrm{RH}$, RT the absorption pattern was found to be higher than at $43 \% \mathrm{RH}$, RT At 75\% RH, RT maximum absorption is shown by urea $10 \% \mathrm{w} / \mathrm{w}$ with $19.42 \%$ absorption \& at $95 \% \mathrm{RH}$, RT with 24.015. Around all the humidity condition the absorption increases with increase in enhancer content. As the humidity increases, there increase in moisture absorption and this increase linear.

At $43 \% \mathrm{RH}, \mathrm{RT}$ all the patches retain their shape at the end of 7 th day, At $75 \%$ RH, RT the urea patches lose their shape on 6th day, while DMSO patches a little sticky to touch. At 95\% RH, RT the urea patches lose their shape on 3rd day while DMSO patches still sticky $\&$ lose their shape on 6th day.

Therefore amongst the enhancers, the patches of UREA \& DMSO are stable for 7 days under different humidity condition.

\subsection{In Vitro Drug Permeation through Cellophane Membrane}

The patches were then subjected to in vitro drug permeation through cellophane membrane and the results obtained are indicated as below;

3.8.1. Table 14. Drug Permeation from PVA Patches 5 $(\mathrm{Mg})$ with Different Urea Concentration

3.8.2. Figure 7. Effect on Drug Permeation from PVA Patches $5(\mathrm{Mg})$ with Different Urea Concentration

3.8.3. Table 15. Drug Permeation from PVA Patches 5 $(\mathrm{Mg})$ with Different DMSO Concentration

\subsubsection{Figure 8. Effect on Drug Permeation from PVA Patches 5 (Mg) with Different DMSO Concentration}

The result obtained is compared with patches without 
Table 14. Drug permeation from PVA patches 5 (Mg) with different urea concentration.

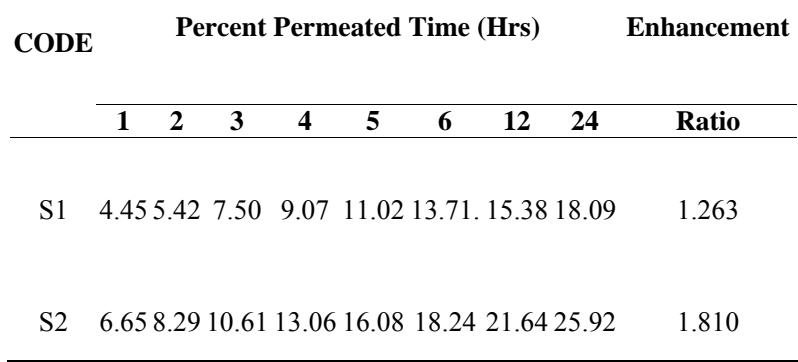

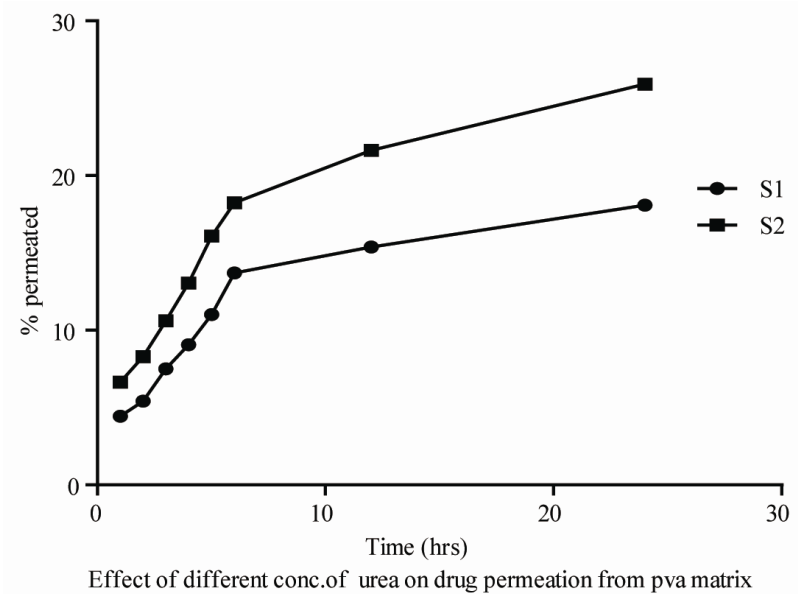

Figure 7. Effect on drug permeation from PVA patches 5 (Mg) with different urea concentration.

Table 15. Drug Permeation from PVA Patches 5 (Mg) with Different DMSO Concentration

CODE Percent Permeated Time (Hrs) Enhancement

\begin{tabular}{cccccccccc} 
& $\mathbf{1}$ & $\mathbf{2}$ & $\mathbf{3}$ & $\mathbf{4}$ & $\mathbf{5}$ & $\mathbf{6}$ & $\mathbf{1 2}$ & $\mathbf{2 4}$ & Ratio \\
\hline $\mathrm{T} 1$ & 2.35 & 3.91 & 5.74 & 7.93 & 9.18 & 11.28 & 13.73 & 16.34 & 1.141 \\
$\mathrm{~T} 2$ & 3.19 & 5.40 & 7.11 & 8.99 & 10.85 & 13.81 & 16.29 & 19.89 & 1.388 \\
\hline
\end{tabular}

penetration enhancers i.e. J3. Amongst the different proportion of urea used, urea at $10 \% \mathrm{w} / \mathrm{w}$ concentration gives maximum drug permeation i.e. of $25.92 \%$ with the enhancement ratio of 1.810 . DMSO also increases the permeation at all concentration. it gives $16.34 \%, 19.89 \%$ of drug permeation for $5 \% \mathrm{w} / \mathrm{w}$ and $10 \% \mathrm{w} / \mathrm{w}$ of DMSO respectively with the enhancement ratio of $1.141 \& 1.388$ respectively. Therefore amongst the various penetration enhancers used in different proportions urea at $10 \% \mathrm{w} / \mathrm{w}$ concentration gives maximum drug permeation i.e. of $25.92 \%$ with the enhancement ratio of 1.810 . Therefore for budesonide in PVA matrix urea $10 \% \mathrm{w} / \mathrm{w}$ is the best

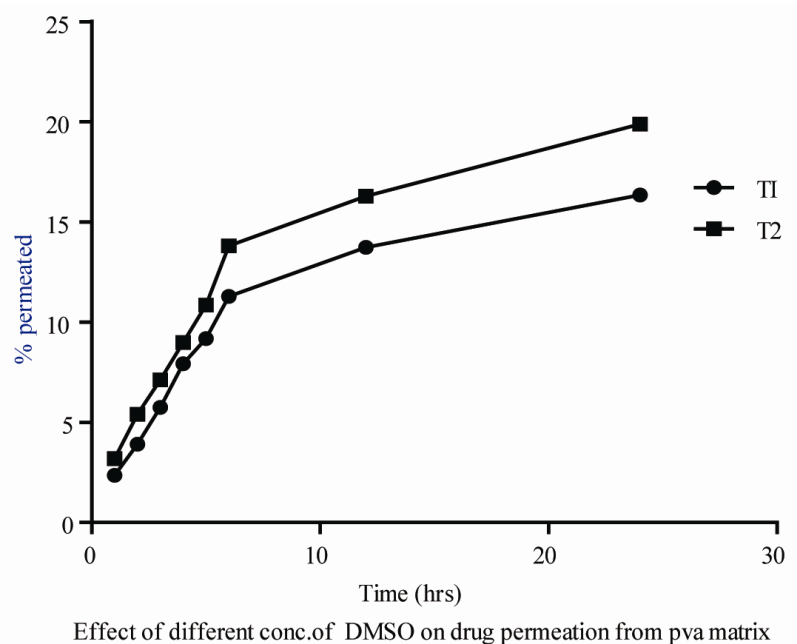

Figure 8. Effect on drug permeation from PVA patches 5 (Mg) with different DMSO concentration.

penetration enhancer.

3.8.5. Table 16. Drug Permeation from EUDRAGIT RL 100 Patches $5(\mathrm{Mg})$ with Different Urea Concentration

3.8.6. Figure 9. Effect of Different Urea Concentration on Drug Permeation from EUDRAGIT RL 100 Patches $5(\mathrm{Mg})$

3.8.7. Table 17. Drug Permeation from EUDRAGIT RL 100 Patches $5(\mathrm{Mg})$ with Different DMSO Concentration

\subsubsection{Figure 10. Effect of Different DMSO Concentration on Drug Permeation from EUDRAGIT RL 100 Patches 5 (Mg)}

The result obtained is compared with patches without penetration enhancers i.e. EB3. Amongst the different proportion of urea used, urea at $10 \% \mathrm{w} / \mathrm{w}$ concentration gives maximum drug permeation i.e. of $27.38 \%$ with the enhancement ratio of 2.01. DMSO also increases the permeation at all concentration. It gives $18.53 \%$, \& $24.10 \%$ of drug permeation for $5 \% \mathrm{w} / \mathrm{w}$ and $10 \% \mathrm{w} / \mathrm{w}$ of DMSO respectively with the enhancement ratio of 1.362 \& 1.772 respectively. Therefore amongst the various

Table 16. Drug permeation from EUDRAGIT RL 100 patches 5 (Mg) with different urea concentration.

CODE Percent Permeated Time (Hrs) Enhancement

\begin{tabular}{cccccccccc}
\cline { 2 - 7 } & $\mathbf{1}$ & $\mathbf{2}$ & $\mathbf{3}$ & $\mathbf{4}$ & $\mathbf{5}$ & $\mathbf{6}$ & $\mathbf{1 2}$ & $\mathbf{2 4}$ & Ratio \\
\hline M1 & 4.92 & 6.94 & 9.42 & 11.22 & 13.51 & 15.93 & 18.07 & 20.65 & 1.515 \\
M2 & 5.43 & 6.96 & 9.59 & 12.33 & 14.90 & 17.76 & 21.90 & 27.38 & 2.013
\end{tabular}




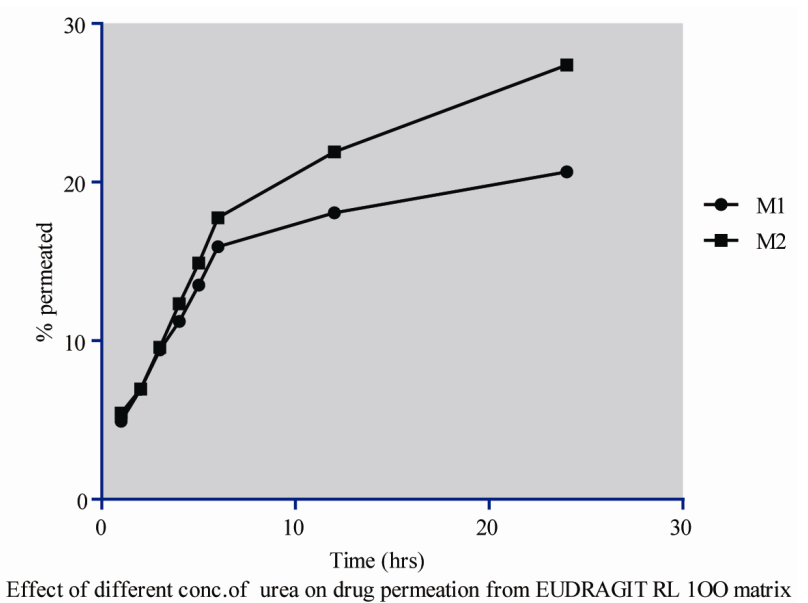

Figure 9. Effect of different urea concentration on drug permeation from EUDRAGIT RL 100 patches 5 (Mg).

Table 17. Drug permeation from EUDRAGIT RL 100 patches 5 (Mg) with different DMSO concentration.

CODE Percent Permeated Time (Hrs) Enhancement

\begin{tabular}{cccccccccc} 
& $\mathbf{1}$ & $\mathbf{2}$ & $\mathbf{3}$ & $\mathbf{4}$ & $\mathbf{5}$ & $\mathbf{6}$ & $\mathbf{1 2}$ & $\mathbf{2 4}$ & Ratio \\
\hline R1 & 4.22 & 5.87 & 7.84 & 9.75 & 11.22 & 13.68 & 15.44 & 18.53 & 1.362 \\
R2 & 5.01 & 6.90 & 9.00 & 12.04 & 15.59 & 17.64 & 20.61 & 24.10 & 1.772 \\
\hline
\end{tabular}

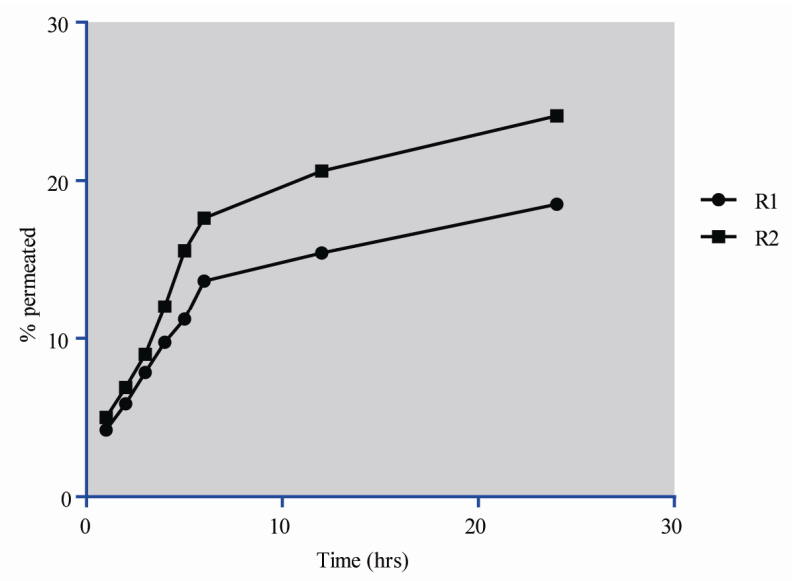

Effect of different conc.of DMSO on drug permeation from EUDRAGIT RL 100 matrix

Figure 10. Effect of different DMSO concentration on drug permeation from EUDRAGIT RL 100 patches 5 (Mg).

penetration enhancers used in different proportions urea at $10 \% \mathrm{w} / \mathrm{w}$ concentration gives maximum drug permeation i.e. of $27.38 \%$ with the enhancement ratio of 2.01 . Therefore for budesonide in EUDRAGIT RL 100 matrix urea $10 \% \mathrm{w} / \mathrm{w}$ is the best penetration enhancer. For both PVA \& EUDRAGIT RL 100 patches urea is the best penetration enhancers than DMSO.

\subsection{Effect of Plasticizers on Films}

The compositions of various films are shown as follows;

\subsubsection{Table 18. Compositions of Patches with Different Plasticizers}

The patches obtained were examined and characterized for the parameter as shown below

\subsubsection{Table 19. Characterization of PVA \& Eudragit RL 100 Patches with Different Plasticizers}

In both the cases the patches formed are uniform with respect to drug content also with increase in the amount of plasticizers the weight of the patch also increase linearly. The thickness was also to be uniform throughout the patch.

\subsection{Moisture Absorption}

\subsubsection{Table 20. Moisture Absorption Study of PVA and EUDRAGIT RL 100 Patches with Different Plasticizers}

At $43 \% \mathrm{RH}, \mathrm{RT}$, the moisture absorption by PVA patches is comparable with patch without the enhancers i.e. J3. The moisture absorption increases with increase in plasticizer content. PEG-400 10\% w/w gives greater absorption than other i.e. $59.87 \%$. Around $75 \% \mathrm{RH}$, RT the absorption pattern is higher than at $43 \% \mathrm{RH}, \mathrm{RT}$. At $75 \%$ RH, RT maximum absorption is shown by PEG-400 10\% w/w with $50.75 \%$ absorption \& at $95 \%$ RH, RT with $59.87 \%$. In all humidity condition the absorption increases with increase in plasticizer content. As the hu-

Table 18. Compositions of patches with different plasticizeers.

\begin{tabular}{ccccc}
\hline CODE & Polymer - mg & $\begin{array}{c}\text { Drug } \\
\text { (mg) }\end{array}$ & $\begin{array}{c}\text { PEG 400 } \\
\text { (mg) }\end{array}$ & $\begin{array}{c}\text { Glyceryl triace- } \\
\text { tate (mg) }\end{array}$ \\
\hline PE1/SO1 & $\begin{array}{c}\text { PVA/Eudragit RL } \\
100-700\end{array}$ & 5 & 35 & -- \\
PE2/SO2 & $\begin{array}{c}\text { PVA/Eudragit RL } \\
100-700\end{array}$ & 5 & 70 & -- \\
GL1/LO1 & $\begin{array}{c}\text { PVA/Eudragit RL } \\
100-700\end{array}$ & 5 & -- & 35 \\
GL2/LO2 & $\begin{array}{c}\text { PVA/Eudragit RL } \\
100-700\end{array}$ & 5 & -- & 70 \\
\hline
\end{tabular}

Table 19. Characterization of PVA \& Eudragit RL 100 patches with different plasticizers.

\begin{tabular}{|c|c|c|c|c|}
\hline CODE & Appearance & $\begin{array}{c}\text { Thickness } \\
\text { (mm) }\end{array}$ & $\begin{array}{c}\text { Weight } \\
\text { (mg) }\end{array}$ & $\begin{array}{c}\text { Drug content } \\
\text { (mg) }\end{array}$ \\
\hline PE1 & Slightly hazy & 0.020 & 28.5 & 0.302 \\
\hline PE2 & Slightly hazy & 0.024 & 31.9 & 0.472 \\
\hline GL1 & $\begin{array}{l}\text { Very Slightly } \\
\text { sticky }\end{array}$ & 0.021 & 31.0 & 0.453 \\
\hline GL2 & $\begin{array}{c}\text { Very Slightly } \\
\text { sticky }\end{array}$ & 0.026 & 34.60 & 0.879 \\
\hline SO1 & Transparent & 0.018 & 26.5 & 0.226 \\
\hline $\mathrm{SO} 2$ & Transparent & 0.020 & 29.2 & 0.335 \\
\hline LO1 & Transparent & 0.019 & 28.1 & 0.430 \\
\hline $\mathrm{LO} 2$ & Transparent & 0.022 & 30.3 & 0.789 \\
\hline
\end{tabular}


Table 20. Moisture absorption study of PVA and EUDRAGIT RL 100 patches with different plasticizers.

\begin{tabular}{|c|c|c|c|c|c|c|c|c|c|}
\hline \multirow[b]{2}{*}{ CODE } & \multicolumn{3}{|c|}{ 43\% RH, RT } & \multicolumn{3}{|c|}{ 75\% RH, RT } & \multicolumn{3}{|c|}{ 93\% RH, RT } \\
\hline & $\begin{array}{l}\text { Wt. of Patch } \\
\text { (mg) }\end{array}$ & $\begin{array}{l}\text { Moist. Absorb. } \\
\text { (mg) }\end{array}$ & $\begin{array}{c}\text { \% absorp- } \\
\text { tion }\end{array}$ & $\begin{array}{l}\text { Wt. of Patch } \\
\text { (mg) }\end{array}$ & $\begin{array}{l}\text { Moist. Absorb. } \\
\text { (mg) }\end{array}$ & $\begin{array}{c}\text { \% absorp- } \\
\text { tion }\end{array}$ & $\begin{array}{l}\text { Wt. of Patch } \\
\text { (mg) }\end{array}$ & $\begin{array}{l}\text { Moist. Absorb. } \\
\text { (mg) }\end{array}$ & $\begin{array}{l}\% \text { absorp- } \\
\text { tion }\end{array}$ \\
\hline PE1 & 28.7 & 5.1 & 17.77 & 28.9 & 12.8 & 44.29 & 28.5 & 15.4 & 54.03 \\
\hline PE2 & 32.4 & 7.4 & 22.83 & 33.1 & 16.8 & 50.75 & 32.9 & 19.7 & 59.87 \\
\hline GL1 & 31.4 & 4.1 & 13.05 & 30.8 & 8.2 & 26.62 & 30.9 & 13.2 & 42.71 \\
\hline GL2 & 34.6 & 7.2 & 20.80 & 34.3 & 13.5 & 39.35 & 34.1 & 17.2 & 50.43 \\
\hline SO1 & 26.2 & 2.2 & 8.39 & 26.7 & 3.4 & 12.37 & 26.5 & 6.9 & 26.03 \\
\hline $\mathrm{SO} 2$ & 29.8 & 3.5 & 11.74 & 29.1 & 6.8 & 23.36 & 29.7 & 9.7 & 32.65 \\
\hline LO1 & 28.4 & 3.5 & 12.32 & 29.1 & 6.9 & 23.71 & 28.6 & 10.8 & 37.76 \\
\hline $\mathrm{LO} 2$ & 30.2 & 4.8 & 15.89 & 29.8 & 7.6 & 25.50 & 30.6 & 11.2 & 36.60 \\
\hline
\end{tabular}

midity increases, there increase in moisture absorption and this increase linear.

At $43 \% \mathrm{RH}, \mathrm{RT}$ all the patches retain their shape at the end of 7th day, At $75 \%$ RH, RT the PEG-400 patches lose their shape on 7th day, while glyceryl triacetate patches a little sticky to touch. At 95\% RH, RT the PEG400 patches lose their shape on 5th day while glyceryl triacetate patches still sticky \& lose their shape on 6th day. At $43 \%$ RH, RT, the moisture absorption by EUDRAGIT RL 100 patches is comparable with patch without the enhancers i.e. EB3. Glyceryl triacetate $10 \%$ w/w absorbs moisture more than any other. At $75 \% \mathrm{RH}$, $\mathrm{RT}$ and at $95 \% \mathrm{RH}, \mathrm{RT}$ the absorption pattern is higher than at $43 \% \mathrm{RH}$, RT At $75 \% \mathrm{RH}$, RT maximum absorption is shown by glyceryl triacetate $10 \% \mathrm{w} / \mathrm{w}$ with $25.50 \%$ absorption \& at $95 \%$ RH, RT with $36.60 \%$. At all humidity condition the absorption increases with increase in enhancer content. As the humidity increases, there increase in moisture absorption and this increase linear. At $43 \%$ RH, RT all the PEG-400 patches retain their shape at the end of 7th day, while glyceryl triacetate patches lost their shape on 6th day. At $75 \%$ RH, RT the PEG-400 patches lose their shape on 6th day, while glyceryl triacetate patches lost their shape on 5th day. At 95\% RH, RT the PEG-400 patches lose their shape on 4th day while glyceryl triacetate patches still sticky \& lose their shape on 3rd day. Therefore amongst the plasticizers, the patches of PEG-400 \& glyceryl triacetate are stable for 7 days under different humidity condition, and selected for final formulation of EUDRAGIT RL 100 \& PVA respectively.

\subsection{In Vitro Drug Permeation}

3.11.1. Table 21. Drug Permeation from PVA Patches with Different PEG-400 and Glyceryl Triacetate Concentration

3.11.2. Table 22. Drug Permeation from Eudragit RL 100 Patches with Different PEG-400 and Glyceryl Triacetate Concentration

3.11.3. Figure 11. Effect of Different Conc. of PEG-400 on Drug Permeation from PVA Matrix
3.11.4. Figure 12. Effect of Different Conc. of Glyceryl Triacetate on Drug Permeation from PVA Matrix

3.11.5. Figure 13. Effect of Different Conc. of PEG-400 on Drug Permeation from EUDRAGIT RL 100 Matrix

\subsubsection{Figure 14. Effect of Different Conc. of Glyceryl Triacetate on Drug Permeation from EUDRAGIT RL 100 Matrix}

The results are compared with patches without plasticizers i.e. J3. The PVA patches with $5 \%$ \& $10 \% \mathrm{w} / \mathrm{w}$ of PEG-400, the permeation increased from $16.50 \%$ to $19.96 \%$. Similarly with glyceryl triacetate the permeation increased from $17.56 \%$ to $23.76 \%$ respectively thus the glyceryl triacetate with $10 \%$ gives maximum permeation hence it is selected for final formulation.

The results are compared with patches without plasticizers i.e. EB3. The EUDRAGIT RL 100 patches with

Table 21. Drug permeation from PVA patches with different PEG-400 and Glyceryl triacetate concentration.

\begin{tabular}{ccccccccc}
\hline & \multicolumn{7}{c}{ Percent permeated } \\
\cline { 2 - 9 } CODE & \multicolumn{7}{c}{ Time in (hrs.) } \\
& \multicolumn{1}{c}{$\mathbf{1}$} & $\mathbf{2}$ & $\mathbf{3}$ & $\mathbf{4}$ & $\mathbf{5}$ & $\mathbf{6}$ & $\mathbf{1 2}$ & $\mathbf{2 4}$ \\
\hline PE1 & 2.39 & 4.52 & 5.96 & 8.20 & 9.75 & 11.21 & 13.81 & 16.50 \\
PE2 & 3.14 & 5.12 & 6.82 & 9.15 & 11.30 & 14.38 & 17.07 & 19.96 \\
GL1 & 3.28 & 4.94 & 6.91 & 8.87 & 10.49 & 12.64 & 14.78 & 17.56 \\
GL2 & 5.01 & 7.13 & 9.02 & 11.26 & 13.78 & 16.40 & 19.78 & $\mathbf{2 3 . 7 6}$ \\
\hline
\end{tabular}

Table 22. Drug permeation from Eudragit RL 100 patches with different PEG-400 and Glyceryl triacetate concentration.

\begin{tabular}{cccccccccc}
\hline & \multicolumn{8}{c}{ Percent permeated } \\
\cline { 2 - 9 } CODE & \multicolumn{8}{c}{ Time in (hrs.) } \\
\cline { 2 - 9 } & $\mathbf{1}$ & $\mathbf{2}$ & $\mathbf{3}$ & $\mathbf{4}$ & $\mathbf{5}$ & $\mathbf{6}$ & $\mathbf{1 2}$ & $\mathbf{2 4}$ \\
\hline SO1 & 4.22 & 6.06 & 8.46 & 10.68 & 12.71 & 15.05 & 17.53 & 20.94 \\
SO2 & 5.01 & 6.29 & 8.79 & 11.07 & 13.72 & 16.39 & 20.19 & $\mathbf{2 4 . 3 3}$ \\
& & & & & & & & \\
LO1 & 4.17 & 5.64 & 7.83 & 9.41 & 11.38 & 13.89 & 16.65 & 19.42 \\
LO2 & 4.82 & 6.56 & 9.07 & 10.99 & 13.46 & 16.30 & 18.97 & 22.54 \\
\hline
\end{tabular}




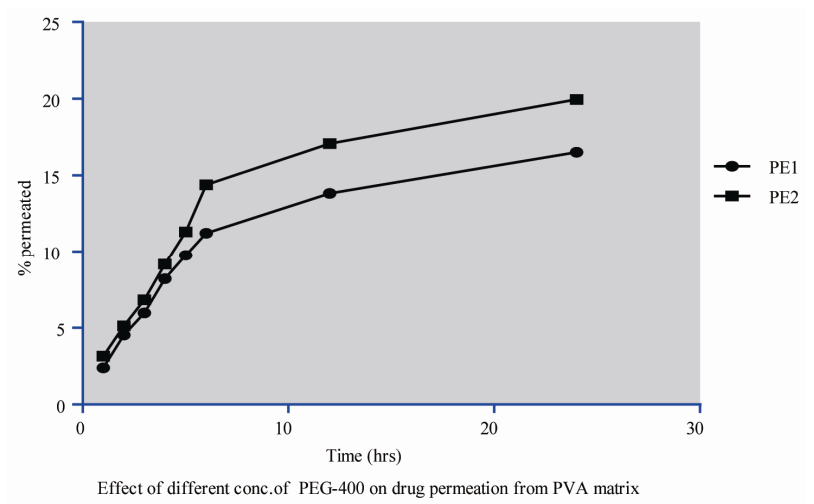

Figure 11. Effect of different Conc. of PEG-400 on drug permeation from PVA matrix.

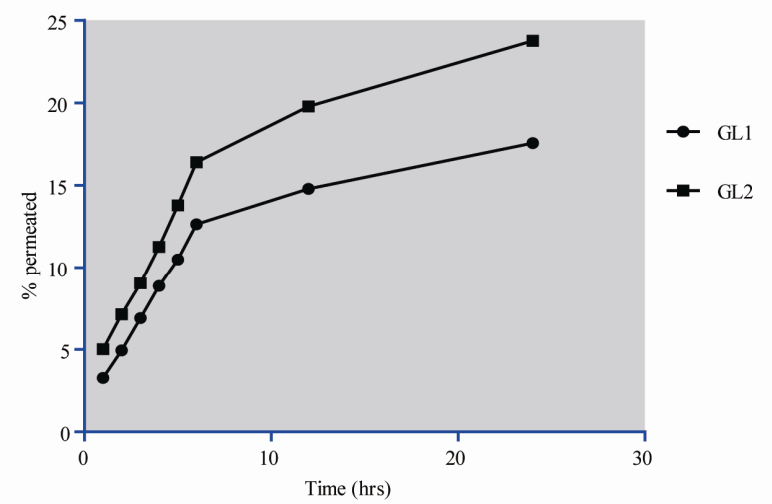

Effect of different conc.of GLYCERYL TRIACETATE on drug permeation from PVA matrix

Figure 12. Effect of different Conc. of Glyceryl Triacetate on drug permeation from PVA matrix.

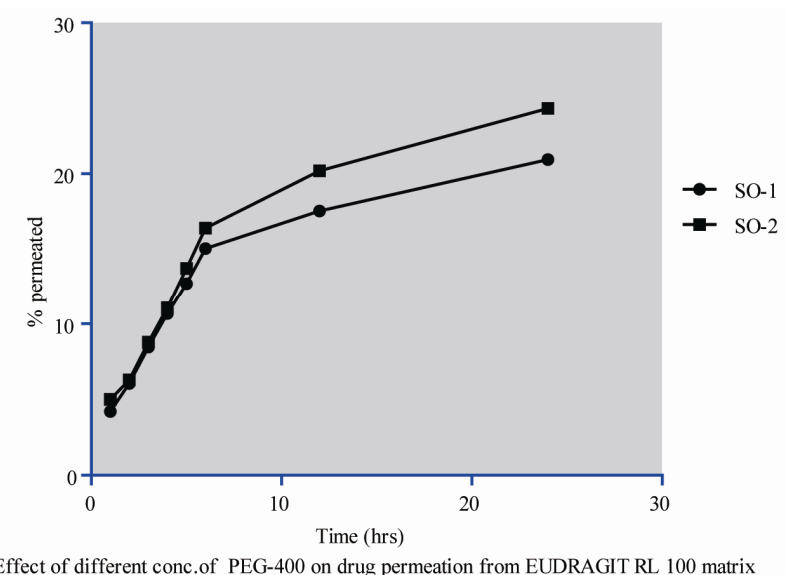

Figure 13. Effect of different Conc. of PEG-400 on drug permeation from EUDRAGIT RL 100 matrix.

$5 \% \& 10 \% \mathrm{w} / \mathrm{w}$ of PEG-400, the permeation increased from $20.94 \%$ to $24.33 \%$. Similarly with glyceryl triacetate the permeation increased from $19.42 \%$ to $22.54 \%$ resp. thus the PEG-400 with $10 \%$ gives maximum permeation

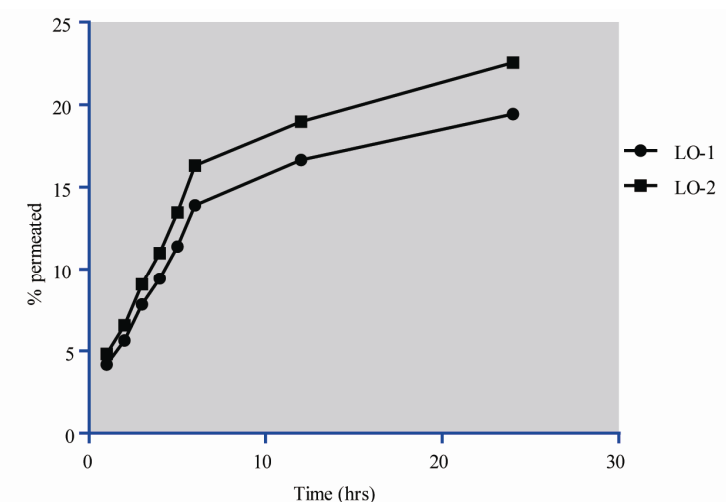

Effect of different conc.of glceryl triacetate on drug permeation from EUDRAGIT RL 100 matrix

Figure 14. Effect of different Conc. of GLYCERYL TRIACETATE on drug permeation from EUDRAGIT RL 100 Matrix.

hence it is selected for final formulation.

\subsection{Final Preparation}

\subsubsection{Preparation and Evaluation of Patch Using Optimum Concentration}

Composition of various film prepared is shown in Table 23.

\subsubsection{Table 23. Composition of Films of PVA and EUDRAGIT RL 100}

The patch obtained was studied and their characterization was done and is listed in following Table 24.

\subsubsection{Table 24. Characterization of PVA and EUDRAGIT RL 100 Patch}

The patch in each group is uniform in drug content and thickness through the patch. And further In Vitro drug permeation, moisture absorption and WVTR studies were carried out.

\subsubsection{Moisture Absorption: Table 25. Moisture Absorption Study of Final PVA and EUDRAGIT RL 100}

Table 23. Composition of films of PVA and EUDRAGIT RL 100.

\begin{tabular}{|c|c|c|c|c|}
\hline CODE & POLYMER (mg) & $\begin{array}{l}\text { DRUG } \\
\text { (Mg) }\end{array}$ & $\begin{array}{c}\text { PLASTISIZER } \\
\text { (Mg) }\end{array}$ & $\begin{array}{l}\text { ENHANCER } \\
\text { (Mg) }\end{array}$ \\
\hline FP-1 & & 5 & GLY.TRI-70 & UREA-70 \\
\hline FE-1 & $\begin{array}{l}\text { EUDRAGIT RL } \\
100-700\end{array}$ & 5 & PEG400- 70 & UREA-70 \\
\hline
\end{tabular}

Table 24. Characterization of PVA and EUDRAGIT RL 100 patch.

\begin{tabular}{llccc}
\hline \multicolumn{2}{l}{ CODEAPPEARANCETHICKNESS } & $\begin{array}{c}\text { WEIGHT } \\
\text { (Mg) }\end{array}$ & $\begin{array}{c}\text { DRUG CON- } \\
\text { TENT (Mg) }\end{array}$ \\
\hline FP-1 & Transparent & 0.019 & 33.4 & 1.971 \\
FE-1 & Transparent & 0.018 & 31.6 & 2.174 \\
\hline
\end{tabular}


Table 25. Moisture absorption study of final PVA and EUDRAGIT RL 100.

\begin{tabular}{|c|c|c|c|c|c|c|c|c|c|}
\hline \multirow[b]{2}{*}{ CODE } & \multicolumn{3}{|c|}{ 43\% RH, RT } & \multicolumn{3}{|c|}{ 75\% RH, RT } & \multicolumn{3}{|c|}{ 93\% RH, RT } \\
\hline & $\begin{array}{l}\text { Wt. of Patch } \\
\text { (mg) }\end{array}$ & $\begin{array}{l}\text { Moist. Absorb. } \\
\text { (mg) }\end{array}$ & $\begin{array}{c}\text { \% absorp- } \\
\text { tion }\end{array}$ & $\begin{array}{l}\text { Wt. of Patch } \\
\text { (mg) }\end{array}$ & $\begin{array}{l}\text { Moist. Absorb. } \\
\text { (mg) }\end{array}$ & $\begin{array}{c}\% \text { absorp- } \\
\text { tion }\end{array}$ & $\begin{array}{l}\text { Wt. of Patch } \\
\text { (mg) }\end{array}$ & $\begin{array}{l}\text { Moist. Absorb. } \\
\text { (mg) }\end{array}$ & $\begin{array}{c}\text { \% absorp- } \\
\text { tion }\end{array}$ \\
\hline FP-1 & 34.4 & 9.8 & 28.48 & 34.9 & 17.9 & 51.28 & 34.1 & 21.3 & 62.46 \\
\hline FE-1 & 31.9 & 6.7 & 21.01 & 31.7 & 12.8 & 40.37 & 32.1 & 15.9 & 49.53 \\
\hline
\end{tabular}

Table 26. WVTR of PVA and Eudragit RL-100 patches.

\begin{tabular}{|c|c|c|c|c|c|c|}
\hline \multirow{2}{*}{ CODE } & \multicolumn{3}{|c|}{$43 \%$ RH, RT } & \multicolumn{3}{|c|}{ 75\% RH, RT } \\
\hline & Wt. of Patch (mg) & Moist. Absorb. (mg) & \% absorption & Wt. of Patch (mg) & Moist. Absorb. (mg) & \% absorption \\
\hline FP-1 & 0.0021 & 0.020 & 0.217 & 0.0151 & 0.022 & 0.882 \\
\hline FE-1 & 0.0391 & 0.017 & 12.76 & 0.145 & 0.018 & 18.86 \\
\hline
\end{tabular}

\subsubsection{Water Vapor Transmission Rate Study (WVTR): Table 26. WVTR of PVA and Eudragit RL-100 Patches}

At all humidity condition the absorption increases. As At all humidity condition the absorption increases. As the humidity increases, there is a increase in moisture absorption and this increases linearly. The patch FP-1 gives maximum absorption at 93\% RH, RT i.e. 62.46 than any other. At $43 \% \mathrm{RH}, \mathrm{RT}$ the PVA patches retain their shape at the end of 7 th day, At $75 \%$ RH, RT the PVA patches lose their shape on 7 th day, At 95\% RH, RT the PVA patches lose their shape on 5th day.

\subsubsection{Drug Permeation through Cellophane Membrane}

3.12.4.1. Table 27. In-Vitro Drug Permeation from EUDRAGIT RL 100 Patches

3.12.4.2. Table 28. In-Vivo Drug Permeation from EUDRAGIT RL 100 Patches

3.12.4.3. Figure 15. Drug Permeation of Patch FP-1

3.12.4.4. Figure16. Drug Permeation of Patch Fe-1 At all humidity condition the absorption increases. As the humidity increases, there increase in moisture absorption and this increase linearly. The patch FE-1 gives maximum absorption at 93\% RH, RT i.e. $49.53 \%$ than any other. At $43 \%$ RH, RT the EUDRAGIT RL 100

Table 27. In-Vitro drug permeation from EUDRAGIT RL 100 patches.

\begin{tabular}{|c|c|c|c|c|c|c|c|c|}
\hline \multirow{3}{*}{ CODE } & \multicolumn{8}{|c|}{ Percent permeated } \\
\hline & \multicolumn{8}{|c|}{ Time ( hrs) } \\
\hline & 1 & 2 & 3 & 4 & 5 & 6 & 12 & 24 \\
\hline
\end{tabular}

$\begin{array}{lllllllll}\text { FE-1 } & 10.94 & 14.25 & 17.60 & 20.85 & 24.26 & 27.71 & 32.07 & 37.25\end{array}$
Table 28. In-Vivo drug permeation from EUDRAGIT RL 100 patches.

CODE

Percent permeated

\begin{tabular}{cccccccc}
\hline \multicolumn{1}{c}{ Time ( hrs) } \\
\hline 1 & 2 & 3 & 4 & 5 & 6 & 12 & 24 \\
\hline
\end{tabular}

$\begin{array}{lllllllll}\text { FE-1 } & 10.94 & 14.25 & 17.60 & 20.85 & 24.26 & 27.71 & 32.07 & 37.25\end{array}$

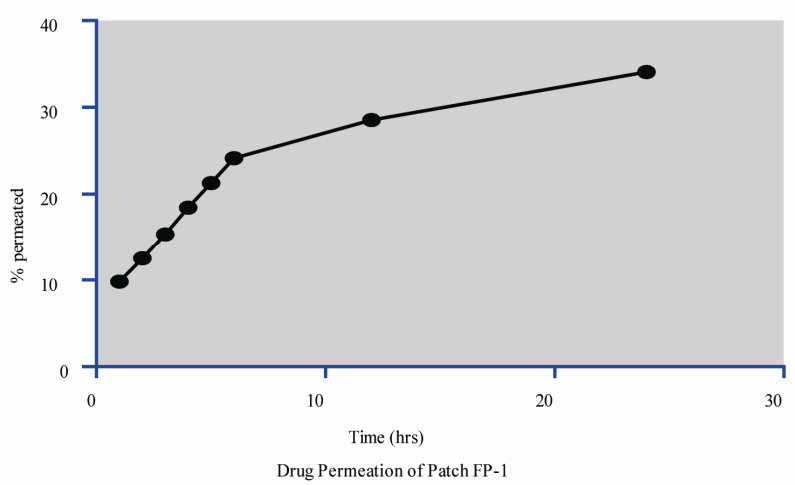

Figure 15. Drug Permeation of Patch FP-1.

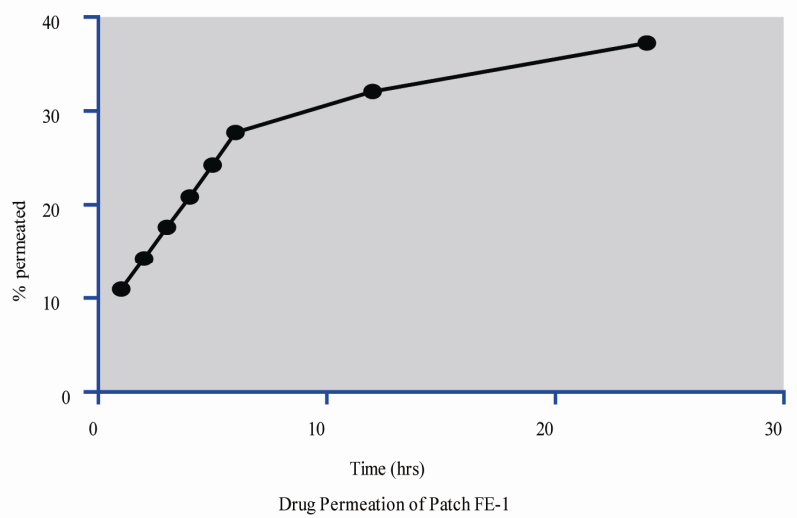

Figure 16. Drug Permeation of Patch Fe-1. 
patches lost their shape at the end of 7 th day, At $75 \% \mathrm{RH}$, RT the EUDRAGIT RL 100 patches lose their shape on 6th day, At 95\% RH, RT the EUDRAGIT RL 100 patches lose their shape on 4th day. In PVA and EUDRAGIT RL 100 patches the water vapor transmission rate was found to be higher at $75 \% \mathrm{RH}$, RT conditions. Therefore at both \% RH, RT condition the PVA and EUDRAGIT RL 100 patches provides the best resistance to water vapor. Therefore, when applied to animals (in further studies) these patches may provide more occlusion to water vapor loss from skin thus making atmosphere beneath the skin more humid that aid in drug permeation.

\section{References}

[1] B. J. Unde, "Pharmacotherapy of Asthma," In: L. L. Brunton, J. S. Lazo and K. L. Parker, Eds., Goodman \& Gilman's, The Pharmacological Basis of Therapeutics, Mcgraw-Hill Medical Publishing Division, New York, 2006, pp. 897-934.

[2] C. M. Spencer and D. McTavish, "Budesonide: A Review of Its Pharmacological Properties and Therapeutic Efficacy in Inflammatory Bowel Disease," Drugs, Vol. 50, No. 5, 1995, pp. 854-872. doi:10.2165/00003495-199550050-00006

[3] R. N. Brogden and D. McTavish, "Budesonide: An Updated Review of Its Pharmacological Properties, and Therapeutic Efficacy in Asthma and Rhinitis," Drugs, Vol. 44, 1998, pp. 375-407. doi:10.2165/00003495-199244030-00007

[4] G. Jeffrey, et al., "Have Done Budesonide Enema for the Treatment of Active, Distal Ulcerative Colitis and Proctitis: A Dose Ranging Study," Current Therapy, Vol. 22, 2005, pp. 23-27.

[5] N. Mygind and T. J. H. Clark, "Topical Steroid Treatment for Asthma and Rhinitis," B. Tindall Inc., London, 1980, pp. 89, 91,152,159,172.

[6] K. Masuyama, et al., "Glucocorticosteroid (Fluticasone propionate) Inhibits Cells Expressing Cytokine mRNA for Interleukin-4 in the Nasal Mucosa in Allergen-Induced Rhinitis," Immunology, Vol. 82, 1994, pp. 192199.

[7] N. S. Chandrashekar and R. H. S. Rani, "Design, Fabrication and Calibration of Modified Franz Diffusion Cell for
Transdermal Diffusion Studies," International Journal of Pharmaceutical Excipients, October-Novem- ber 2005, pp. 104-106.

[8] J. Eliassaf, "Detection of Small Quantity of Poly(Vinyl Alcohol) in Poly(Vinyl Chloride) Resins," Polymer Letters, Vol. 16, 1972, pp. 225-235.

[9] S. P. Gupta and S. K. Jain, "Development of MatrixMembrane Transdermal Drug Delivery System for Atenolol," Drug Delivery, Vol. 11, No. 5, 2004, pp. 281286. doi:10.1080/10717540490493943

[10] P. R. P. Verma and S. S. Iyer, "Transdermal Delivery of Propranolol Using Mixed Grades of Eudragit: Design and in Vitro and in Vivo Evaluation," Drug Development and Industrial Pharmacy, Vol. 26, No. 4, 2000, pp. 471-476. doi:10.1081/DDC-100101257

[11] R. Krishna and J. K. Pandit, "Transdermal Delivery of Propranolol," Industrial Pharmacy, Vol. 20, No. 15, 1994, pp. 2459- 2465.

[12] P. Arora and B. Mukherjee, "Design, Development, Physicochemical and in Vitro and in Vivo Evaluation of Transdermal Patches Containing Diclofenac Diethylammonium Salt," Journal of Pharmaceutical Sciences, Vol. 91, 2002, pp. 2076-2089. doi:10.1002/jps. 10200

[13] United States Pharmacopoeia, "Physical Tests <711> Dissolution," Vol. 24, 2000, pp. 1941.

[14] M. Siewert, J. Dressman, C. K. Brown and V. P. Shah, "FIP/AAPS Guidelines to Dissolution/in Vitro Release Testing of Novel/Special Dosage Forms," AAP Spharmscitech, Vol. 4, No. 1, 2003, pp. 1-10.

[15] C. Valenta and B. G. Auner, "The Use of Polymers for Dermal and Transdermal Delivery," European Journal of Pharmaceutical Sciences, Vol. 58, No. 2, 2004, pp. 279289.

[16] P. Costa, "Modeling and Comparison of Dissolution Profiles," European Journal of Pharmaceutical Sciences, Vol. 13, No. 2, 2001, pp. 123-133. doi:10.1016/S0928-0987(01)00095-1

[17] J. Singh and H. I. Maibach, "Irritancy of Topical Chemicals and Transdermal Delivery Systems," Drug Pharmaceutical Sciences, Vol. 1, 2001, pp. 281-296.

[18] A. C. Calpena, E. Escribano and H. San Martin, "Influence of Formulation on the in Vitro Transdermal Penetration of Sodium Diclofenac," Arzneimittle Forschung Drug Research, Vol. 49, No. 11, 1999, pp. 1012-1017. 